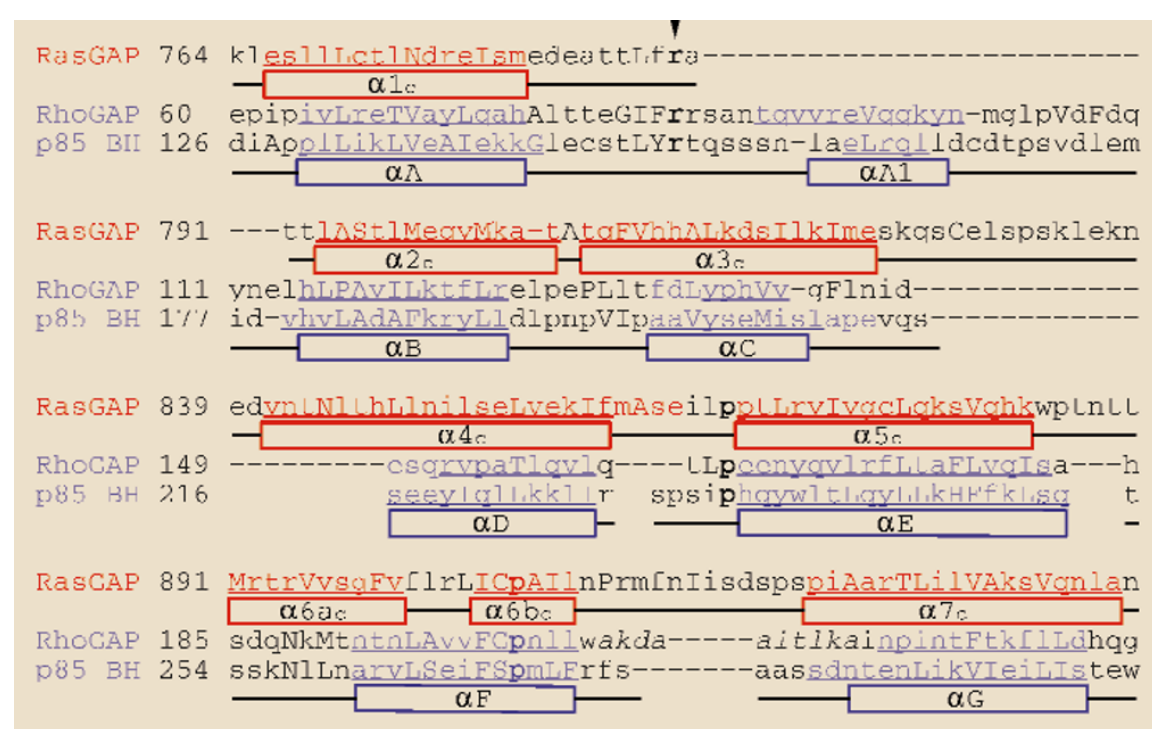

Figure $1 \mathrm{~A}$ structurally based sequence alignment of comparable regions of the rasGAP domain from p120GAP (PDB code: 1wer), the rhoGAP domain of p50rhoGAP (PDB code: 1rgp) and the BH rhoGAP-like domain of the p85 subunit of phosphatidylinositol-3-OH kinase (PDB code: 1pbw). The computer program COMPARER ${ }^{78}$ provided the alignment. $\alpha$ Helices are underlined and shown in red (rasGAP) or blue (rhoGAP, p85 BH); $3_{10}$ helices are not underlined. The nomenclature for helices is as originally defined ${ }^{2,6,4}$. Residues labelled in upper-case lettering are inaccessible to solvent. The position of Arg 789 (rasGAP)/Arg 85 (rhoGAP)/Arg 151 (p85 BH) is indicated by the arrow. Residues between helix $\alpha F$ and $\alpha G$ are disordered in the rhoGAP structure (in italics).

p85 $\mathrm{BH}$ (where $\mathrm{BH}$ indicates a BCRhomology domain), has also been determined $^{6}$. The release of the coordinates for the rhoGAP and rasGAP domains allowed us to compare their structures. We found that the rhoGAP and rasGAP domains are clearly related (my Fig. 1 and Fig. 1 in the letter below by Rittinger et al.) and must have derived from the same ancestral protein.

To see the equivalence of the structures more clearly (in Fig. 1 of Rittinger et al.), place your finger on the top of helix $\mathrm{F}$ (of rhoGAP) and imagine pushing it away from you and down so that it lies more nearly parallel to helix $G$ and between helices $G$ and $\mathrm{E}$ (helices $\mathrm{E}$ and $\mathrm{A}$ then move to the left). The rasGAP and rhoGAP sequences (in my Fig. 1) are only 6\% identical, whereas rasGAP and $\mathrm{p} 85 \mathrm{BH}$ are $13 \%$ identical (the two rhoGAP-like domains, whose similarity was identified at the sequence level, have a $17 \%$ sequence identity).

In the transition-state-analogue complexes of both Ras-rasGAP ${ }^{3}$ and Rho-rhoGAP ${ }^{5}$, helix $\alpha 6 / \alpha \mathrm{F}$ interacts with the switch II loop on Ras/Rho. This interaction helps to stabilize the conformation of the switch II loop which provides residue Gln 61 ( $G \ln 63$ in Rho) for the active site. The large structural shift in the relative orientation of helix $\alpha 6 / \alpha \mathrm{F}$ of rasGAP/rhoGAP may have occurred to compensate for the different structures of the switch II loop in Ras and Rho.

This equivalence in structure points to a parallel evolution by Ras and Rho G proteins and their GAPs.

\section{Benjamin Bax}

Department of Crystallography, Birkbeck College,

Malet Street, London WC1E 7HX, UK

e-mail:b.bax@mail.cryst.bbk.ac.uk

\footnotetext{
. Ahmadian, M. R., Stege, P., Scheffzek, K. \& Wittinghofer, A. Nature Struct. Biol. 4, 686-689 (1997).

2. Scheffzek, K., Lautwein, A., Kabsch, W., Ahmadian, M. R. \& Wittinghofer, A. Nature 384, 591-596 (1996)

3. Scheffzek, K. et al. Science 277, 333-338. (1997)

4. Barrett, T. et al. Nature 385, 458-461 (1997).

5. Rittinger, K., Walker, P. A., Eccleston, J. F., Smerdon, S. J. \& Gamblin, S. J. Nature 389, 758-762 (1997).

6. Mussachio A., Cantley, L. C. \& Harrison, S. C. Proc. Natl Acad. Sci. USA 93, 14373-14378 (1996).

. Sali, A., \& Blundell, T. L. J. Mol. Biol. 212, 403-428 (1990)

8. Zhu, Z.-Y., Sali,A. \& Blundell, T. L. Protein Engng 5, 43-51 (1992).
}

The Ras superfamily of monomeric GTPase proteins comprises six subfamilies, each with specific cellular functions, but all these subfamilies share a common fold and a common mechanism for hydrolysing GTP. Unlike their cognate guanine-nucleotidebinding (G) proteins, GTPase-activating proteins (GAPs) specific for the Rho or Ras subfamilies show no significant homology at the level of protein sequence. However, we have now noticed a structural similarity which suggests that, like their $G$ proteins, these GAPs have evolved from a common ancestor.

Small G proteins belonging to the Ras superfamily act as signalling molecules con-

\section{Support for shared ancestry of GAPs}

trolling a wide range of biological processes. Crucial to their signalling function is the conformational switching that takes place between their active, GTP-bound and inactive, GDP-bound forms ${ }^{1}$. They have a slow, intrinsic rate of GTP hydrolysis which is substantially accelerated by GAPs specific to each subfamily. Crystallographic coordinates for rhoGAP and rasGAP are now available ${ }^{2,3}$ and we have compared them using the program LSQMAN (ref. 4).

Superposition of the two GAPs shows that they share a core structure made up of seven $\alpha$-helices (Fig. 1). The $\alpha$-helices making up this core pack against each other in a related, but not identical, fashion. However, the automated alignment puts the catalytic arginine residues (R85 in rhoGAP and R789 in rasGAP) into approximately the same position and, by implication, locates the active sites of the bound $\mathrm{G}$ proteins.

It is particularly interesting to note that in both structures the catalytic arginine residue is located on a surface loop and is preceded by two bulky, hydrophobic residues (isoleucine and phenylalanine at residues 83 and 84 , respectively, in rhoGAP, and leucine and phenylalanine at positions 787 and 788, respectively, in rasGAP). These residues are held in a hydrophobic clamp made up of residues from the $\mathrm{B}(2 \mathrm{c}), \mathrm{E}(5 \mathrm{c})$ and $\mathrm{F}(6 \mathrm{c})$ helices that act to anchor the catalytic arginine loop to the core of the domain.

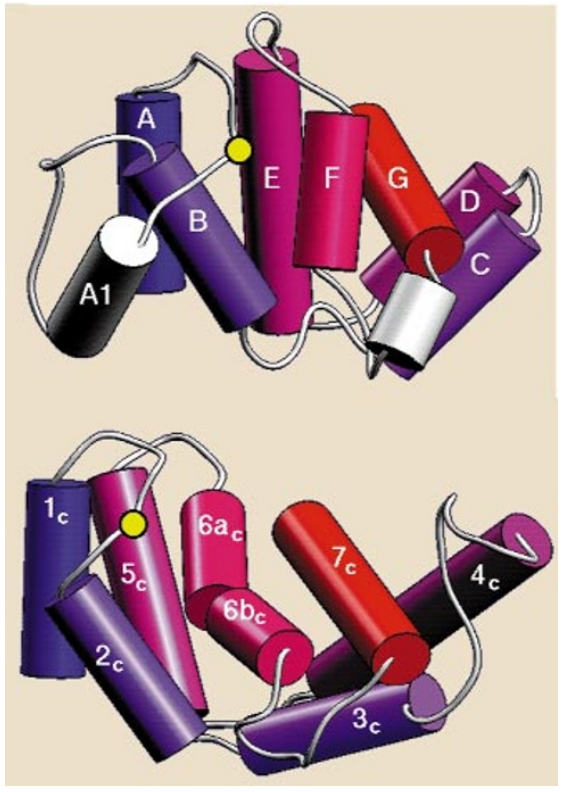

Figure 1 The common core structures of the catalytic domains of p50rhoGAP (top) and p120rasGAP (bottom), as aligned by the computer program LSQMAN, in helical-tube representation. Helical segments forming the core structure (helices $A-G$ and $1 \mathrm{c}$ to $7 \mathrm{c}$ in rhoGAP and rasGAP, respectively) are coloured from blue at their amino termini to red at their carboxy termini. Helical insertions that do not form part of the core motif are grey. The positions of the catalytic arginines R85(rhoGAP) and R789(rasGAP) are indicated by yellow circles. 
The published structures of the transition-state-analogue complexes of RhoGDP. $\mathrm{AlF}_{4}^{-}$/p50rhoGAP (ref. 5) and RasGDP.AlF $\mathrm{Al}_{3} / \mathrm{p} 120$ rasGAP (ref. 6) show that the two GAP domains bind their respective $G$ proteins in related but distinct ways. rhoGAP binds to Rho primarily through a surface made up by the helices B and F, whereas rasGAP binds to Ras through the antiparallel helices $6 \mathrm{c}$ and $7 \mathrm{c}$.

These differences in G-protein-binding surfaces arise because of the different spatial relationship between helices $\mathrm{A}-\mathrm{B}$ and $\mathrm{E}-\mathrm{F}$ of rhoGAP and $1 c-2 c$ and $5 c-6 c$ of rasGAP. Relative to the rhoGAP helices $\mathrm{E}$ and $\mathrm{F}$, the carboxy terminal (or top half) of $5 \mathrm{c}$ bends back into the plane of the page while the whole of $6 c$ rotates further in a similar direction.

Nonetheless, both GAP domains present their catalytic arginine residues to the active sites of the $G$ protein in very similar ways, which is a consequence of the equivalence in function of the arginine loop in these two systems. It will be intriguing to see whether other GAPs associated with the Ras superfamily contain the same core structure, obey the same topology, and make similar use of hydrophobic clamping to orient the catalytic arginine loop.

Katrin Rittinger, William R. Taylor,

Stephen J. Smerdon, Steven J. Gamblin

Structural Biology Group,

National Institute for Medical Research,

The Ridgeway, London NW7 1AA, UK

e-mail:s-smerdo@anika.nimr.mrc.ac.uk

1. Boguski, M. S. \& McCormick, F. Nature 366, 643-654 (1993).

2. Barrett, T. et al. Nature 385, 458-461 (1997).

3. Scheffzek, K., Lautwein, A., Kabsch, W., Ahmadian, M. R. \& Wittinghofer, A. Nature 384, 591-596 (1996)

4. Kleywegt, G. J. \& Jones, T. A. Meth. Enzymol. 277, 525-546 (1997).

5. Rittinger, K., Walker, P. A., Eccleston, J. F., Smerdon, S. J. \& Gamblin, S. J. Nature 389, 758-762 (1997)

6. Scheffzek, K. et al. Science 277, 333-338 (1997).

\section{Meningitis bacterium is} viable without endotoxin

The outer membrane of Gram-negative bacteria contains lipopolysaccharide (LPS) as its outer monolayer. This is anchored to the membrane by lipid A, which is responsible for LPS's activity as an endotoxin ${ }^{1}$. In Escherichia coli, conditionally lethal mutants have been reported for the genes involved in the early steps of lipid A biosynthesis $^{2}$, suggesting that this part of the LPS molecule is essential for bacterial growth. However, we have isolated a mutant of Neisseria meningitidis which is viable in spite of an early block in lipid A biosynthesis that causes a loss of endotoxin activity.

The protein LpxA is responsible for adding the $\mathrm{O}$-linked $3-\mathrm{OH}$ fatty acid to
$\mathrm{UDP}-N$-acetylglucosamine, which is the first committed step in the lipid A biosynthesis pathway ${ }^{3,4}$. The E. coli and $N$. meningitidis LpxA proteins differ in their specificity, preferring $3-\mathrm{OH} \mathrm{C} \mathrm{C}_{14}$ and $3-\mathrm{OH}$ $\mathrm{C}_{12}$ fatty acyl chains, respectively ${ }^{5,6}$.

We constructed a hybrid $I_{p} x A$ gene in which the meningococcal N-terminal part was replaced by the corresponding part of E. coli $1 p x A$. Plasmid pHBK30, carrying this hybrid gene and an upstream kanamycinresistance cassette, was used for allelic replacement of the wild-type lpxA gene on the chromosome of meningococcal strain H44/76. Lipopolysaccharide of the H44/76[pHBK30] mutant and the wildtype strain was compared by TricineSDS-PAGE followed by silver staining for carbohydrates (Fig. 1a). No LPS could be detected in the hybrid derivative by this method, even when higher amounts of cell lysates were loaded on the gel.

A panel of LPS and monoclonal antibodies specific for outer-membrane proteins (OMPs) was tested in a whole-cell enzyme-linked immunosorbent assay $(\text { ELISA })^{7,8}$. The mutant strain did not bind any of the LPS-specific antibodies (either immunotype-specific or broadly crossreactive), whereas the OMP-specific antibodies showed similar binding patterns for mutant and wild type. This apparent OMP similarity was confirmed when outer-membrane complexes (OMCs) of H44/76[pHBK30] and H44/76 were isolated by sarkosyl extraction and analysed by SDS-PAGE (Fig. 1b). Both strains show equal amounts of the class 1, 3 and 4 principal OMPs.

As LPS of H44/76[pHBK30] could not be detected, it was not clear whether it was present at all. Therefore, the mutant and wild-type strain were tested in a chromogenic Limulus (LAL) assay ${ }^{9}$ for endotoxin, using the QCL-1000 kit from BioWhittaker. The results of the LAL assay on cell suspensions showed no significant endotoxin activity for H44/76[pHBK30] over meningococcal medium ( 0.3 and 1.7 endotoxin units per millilitre, respectively), in contrast to $21.7 \times 10^{4}$ endotoxin units per millilitre for the wild type.

Although H44/76[pHBK30] is viable, it had a reduced growth rate. When grown overnight on GC agar plates, the mutant strain produced much smaller colonies. In liquid medium, the doubling time during exponential growth was about 50\% longer than in the wild-type strain H44/76. The morphology of H44/76[pHBK30] and its parent strain was examined by electron microscopy of ultrathin sections (Fig. 1c). The ultrastructure of the outer membrane could be clearly discerned in the LPSdeficient mutant and was not visibly altered.

Strain H44/76[pHBK30] is viable and possesses an outer membrane, despite lacking detectable LPS. It seems likely that the

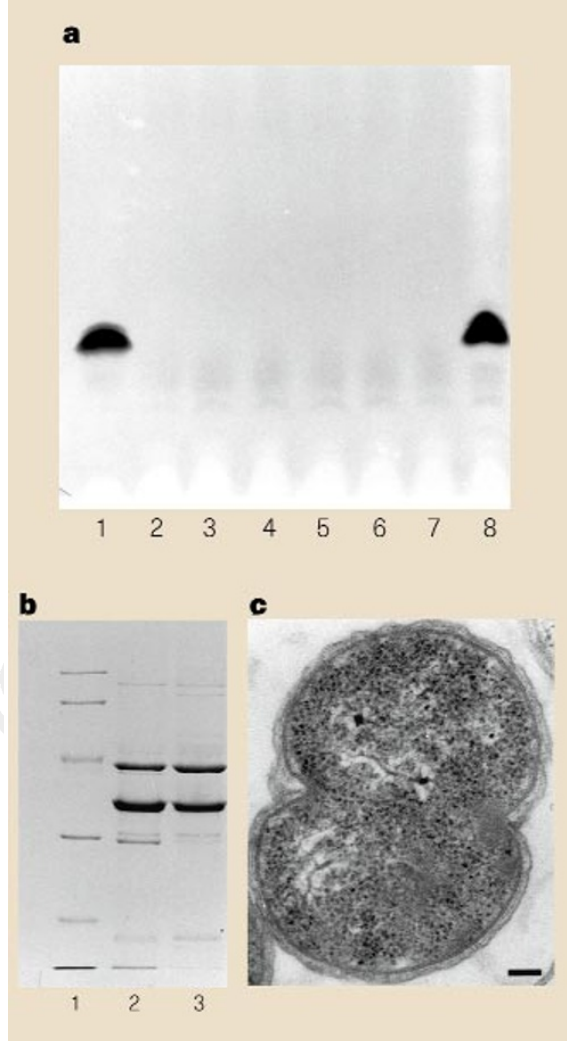

Figure 1 Analysis of Neisseria meningitidis outer membrane. a, Silver-stained Tricine-SDS-PAGE LPS gel of proteinase K-treated whole-cell lysates of $\mathrm{H} 44 / 76$ wild type (lanes 1 and 8) and six independent kanamycin-resistant transformants with pHBK30 (lanes 2-7). b, SDS-PAGE of outer membrane proteins from H44/76[pHBK30] (lane 2) and H44/76 wild type (lane 3 ); lane 1 contains relative molecular mass markers of 94K, 67K, 43K, 30K, $20.1 \mathrm{~K}$ and $14.4 \mathrm{~K}$. c, Electron micrograph of a thin section of $\mathrm{H} 44 / 76[\mathrm{pHBK} 30]$, showing normal appearance of the cell envelope with outer membrane, peptidoglycan layer within the periplasmic space, and inner membrane. Scale bar, $100 \mathrm{~nm}$.

hybrid $l p x A$ gene is inactive, either because of disrupted transcription/translation in our construct, or otherwise because the hybrid protein produced lacks enzymatic activity. We constructed an $I p x A$-knockout mutant by inserting a kanamycin-resistance cassette into the BstEII site located at position 293 in the $l_{p x A}$ gene of plasmid pLA21 (a pUC18 derivative with a 2.1 kilobase lpxD-fabZ-lpxA insert). The resulting plasmid pLAK33 was linearized and transformed to strain H44/76, with selection for kanamycin resistance. The resulting colonies showed the same growth properties as the H44/76[pHBK30] mutant. In whole-cell ELISA, the lpxA-knockout mutant did not bind any of the LPS-specific monoclonal antibodies.

The absence of LPS was further confirmed by gas chromatography/mass spectrometry analysis of fatty acids present in OMC preparations, which showed that the lipid A-specific 3-OH $\mathrm{C}_{12}$, whose addition 